\title{
Cosmic ray ionization effect in the atmosphere during the maximal GLE05 - on 23.02.1956
}

\author{
Peter I.Y. Velinov \\ Institute for Space Research and Technology, Bulgarian Academy of Sciences \\ Acad. G. Bonchev St., Bl. 1, 1113 Sofia, Bulgaria \\ E-mail: pvelinov@bas.bg
}

Yury V. Balabin ${ }^{1}$, Evgeny A. Maurchev
Polar Geophysical Institute, Apatity, Russian Academy of Sciences
26a, Academgorodok St.,184209 Apatity, Russian Federation
E-mail: balabinepgia.ru , E-mail: maurchevepgia.ru

The ground level enhancements (GLEs) due to solar cosmic rays (SCRs) are of significant astrophysical, cosmophysical and geophysical interest for a number of planetary processes on the Earth. In the present work the ionization rate profiles in the Earth's atmosphere during the outstanding ground level enhancement GLE05 on 23 February 1956, the largest one in the entire history of observations of SCRs, are obtained. The contribution of the galactic cosmic rays (GCRs) is also taken into account. The ionization in the atmosphere is calculated using the energetic spectra of solar protons, GCRs and the corresponding atmospheric cascade simulation. The spectra of solar cosmic rays are derived on the basis of ground-based measurements with neutron monitors. This method is based on solving the inverse problem - reconstruction of SCR spectrum on the boundary of the magnetosphere. The contemporary magnetosphere model "Tsyganenko 01 and 03 " is used in this method. The calculation of asymptotic cones (AK) held with increments of $0.001 \mathrm{GV}$ in the range of 1-20 GV. The aforementioned method is developed in the Polar Geophysical Institute (in Apatity) of the Russian Academy of Sciences. Recent simulations with RUSCOSMICS software package (based on GEANT4 program of CERN) are carried out. The energy deposit of solar protons and GCRs in the atmosphere is received. The ion production rate profiles in the stratosphere and troposphere (the region $0-40 \mathrm{~km}$ ) are calculated for geomagnetic cut-off rigidities 1, 2, 3 and $5 \mathrm{GV}$ (i.e. polar, subpolar, higher and middle latitudes, respectively). The so obtained ionization profiles are compared and thoroughly discussed. Implementations of these results are also debated. The obtained results are important for the improvement of the recent models of cosmic ray induced ionization in the whole atmosphere, for the determination of electron and ion density in the middle and lower atmosphere (troposphere), for the various atmospheric processes (as for instance in atmospheric chemistry and physics ozone production, global electric circuit between the ground and ionosphere), for the studies of solar-terrestrial influences on space weather and space climate) and for many other applications.

35th International Cosmic Ray Conference - ICRC2017

10-20 July, 2017

Bexco, Busan, Korea

\section{${ }^{1}$ Speaker}




\section{Introduction}

The ground level enhancements (GLEs) are caused by the relativistic solar energetic particles on the ground based detectors - mainly neutron monitors but also muon telescopes and ionization chambers (before the International Geophysical Year IGY, 1957-1958). Really the relativistic solar energetic particles (SEPs) are solar cosmic rays (SCRs) and they consist basically of protons (more than 90\%). Their energies usually are in the $\mathrm{GeV}$ range. Rarely SCRs can exceed $15-20 \mathrm{GeV}[1,2]$. The official database of Neutron Monitor count rates during GLEs is situated on the website http://gle.oulu.fi/. In the present study we consider the outstanding ground level enhancement GLE05 on 23 February 1956, the largest one in the entire history of observations of solar cosmic rays. This maximal GLE has been extensively studied for a period more than 60 years $([3,4]$ and reference therein). The GLE05 was caused by a large solar flare with importance of $3+$ (or 3B) that occurred at 03:31 UT in the active region with heliographic coordinates $25^{\circ} \mathrm{N}$, $85^{\circ} \mathrm{W}$.

The event on 23 February 1956 exerted essential influence on the development of all sciences of solar-terrestrial physics and physics: solar physics, cosmic rays (CRs), magnetosphere, ionosphere, etc. In this work we will examine the final effect of SCRs - the induced ionization in the Earth's atmosphere during GLE05.

\section{Earlier results for enhanced ionization in ionosphere during GLE05}

Over 50 years ago were made calculations of ionization profiles $q(h)$ in the lower ionosphere $(50-100 \mathrm{~km})$ due to the penetration of relativistic SEPs during the GLE05 on February 23, 1956 [5-9]. In the atmosphere cosmic particles enter in electromagnetic and nuclear interactions with the substance of the air. The first results for $q(h)$ profiles [5-9] were obtained without accounting the nuclear interactions between the secondary cosmic ray particles and the Earth's atmosphere, which is quite acceptable for these altitudes. In the ionospheric D-region (50$100 \mathrm{~km}$ ) the predominant interactions are the electromagnetic due to the small atmospheric depth (less than $1 \mathrm{~g} / \mathrm{cm}^{2}$ ) for the cosmic ray particles [7].

\section{Previous computations for ionization in strato-troposphere during GLEs}

In the altitudinal range $0-40 \mathrm{~km}$ the induced ionization is a result from the impact of the electromagnetic, muon and hadronic components of the secondary galactic and solar cosmic rays (GCRs and SCRs) on the terrestrial atmosphere. That's why after receiving the initial results [59] were made calculations taking into account all nuclear reactions [10-12]. One possible tool is based on Monte Carlo simulation of the cascade process in the atmosphere, precisely the energy deposit and afterwards the estimation of ion pair production. CORSIKA (Cosmic Ray Simulations for Kaskade) code is one of the most widely used in the last years simulation code $[11,12]$. By means of CORSIKA 6.52 program using FLUKA 2006 and QGSJET II hadronic interaction subroutines it was determined the induced ionization in atmosphere during some important GLEs, f.e.: GLE59 on Bastille day event 14 July, 2000 [13, 14]; GLE69 on 20 January 2005 [15] - the second in intensity after GLE05; GLE70 on 13 December 2006 in solar minimum [16, 17], etc. Now we will continue these studies. The ion production rate in the atmosphere during the greatest GLE05 on 23 February 1956 will be calculated. This induced ionization is due to the influence of the all three secondary $\mathrm{CR}$ components: electromagnetic, muon and hadronic. 


\section{Determination spectra of galactic and solar cosmic rays}

First we need to determine the spectra of GCRs and SCRs. The energy spectrum of GCRs, established by GOST USSR, has been used for the calculation of ionization in the atmosphere. The GOST standard defines the GCR spectrum from $200 \mathrm{MeV}$ to hundreds GeV. At the same time it considers the spectrum changes due to solar activity. It is taken into account every specific case by calculation the ionization profiles due to GLEs.

Usually an approximate formula for GCR intensity $I$ is $[5,7]$ :

$$
I_{\mathrm{N}}(E) \propto 1.8 E^{\gamma} \text { nucleons } / \mathrm{cm}^{2} \mathrm{~s} \mathrm{sr}
$$

where $E$ is the total particle energy per nucleon in $\mathrm{GeV}$ and $\gamma=-2.7$ is the spectral index. It is known that the intensity of GCRs is modulated by the solar activity and depends from geomagnetic latitude. The specific formula (1) refers to the middle latitudes - geomagnetic cutoff rigidity $\approx 5 \mathrm{GV}$ and an average solar activity.

The differential spectrum of SCRs generally has the following description $[6,7,10]$ :

$$
D(E)=\mathrm{k} E^{-\gamma}
$$

The values of $\mathrm{k}$ and $\gamma$ are different (usually $\gamma$ is changed from -4 to -6 ) at different moments of development of SPE event, because the proton flux (when reaches the Earth) has variations in different energy intervals and at different moments.

For account the GLE influence on the ionization of the atmosphere are used the spectra of SCRs, obtained by the method developed in the Polar Geophysical Institute - PGI (Apatity) of the Russian Academy of Sciences (RAS). This method is based on solving the inverse problem the reconstruction of the SCR spectrum on the boundary of the magnetosphere on the basis of ground-based measurements with neutron monitors. The method is described in details in [1, 4].

The contemporary magnetosphere model "Tsyganenko 01 and 03 " is used in this method. The calculation of asymptotic cones (AK) held with increments of $0.001 \mathrm{GV}$ in the range of 1-20 GV. The knowledge of AK is not too important for GCRs, because the GCR flux is close to isotropic. At the same time, anisotropy in the SCRs comes to the initial stage 1, so that an accurate calculation of AK, especially in the penumbra region, leads to a more exact solution of the inverse problem. The developed method was applied in PGI (Apatity) - RAS to three dozen GLE events and the results of solutions were verified according to direct measurements on sounding balloons (in the stratosphere) and from satellite data.

The calculated by us primary differential spectra - the dependence of the intensity of particles $\left(\mathrm{m}^{-2} \mathrm{~s}^{-1} \mathrm{sr}^{-1} \mathrm{MeV}^{-1}\right)$ from their energy $(\mathrm{MeV})$ for GCRs and SCR protons are presented in Fig. 1. Based on that figure we can make the following analysis.

During the GLE events SCR flux exceeds GCRs 2-3 orders of magnitude at energies 0.5$5 \mathrm{GeV}$, however, due to high values of the exponent gamma $\gamma(\gamma \approx-4--6$ for SCRs (2), whereas $\gamma=-2.7$ for the GCRs (1)) SCR flux at energies above $20 \mathrm{GeV}$ becomes less than GCRs. And the steeper fall the SCR spectrum (greater $\gamma$, i.e. softer spectrum (2)), the greater is the ionization effect in the upper and middle atmosphere. In our example (GLE05 event) this is not noticeable, because this event was one of the hardest according to the CR spectra: in this case the intensity of relativistic solar particles significantly exceed the intensity of GCR to $\sim 15-20 \mathrm{GeV}$. While in the other cases of GLEs usually already at $5 \mathrm{GeV}$ the SCR flux becomes less than GCR flux [14]. 


\section{Modeling the primary CR proton transport trough the Earth's atmosphere}

When CRs enter the Earth's atmosphere, they interact with its atoms and molecules. As a result nuclei, fissure and many secondary particles are formed. By investigating particle cascades, we can obtain information on particle generation and propagation, on primary cosmic ray fluxes, and estimate the rate of ion production rate in the middle and lower atmosphere [18-20].

With the start of computers era has become to actively develop the investigation method of the propagation of CR in a matter by means the Monte Carlo method. In the works $[18,19]$ is developed a software package RUSCOMICS, including a detector models of various types, as well as the primary CR propagation through the Earth's atmosphere model to get information

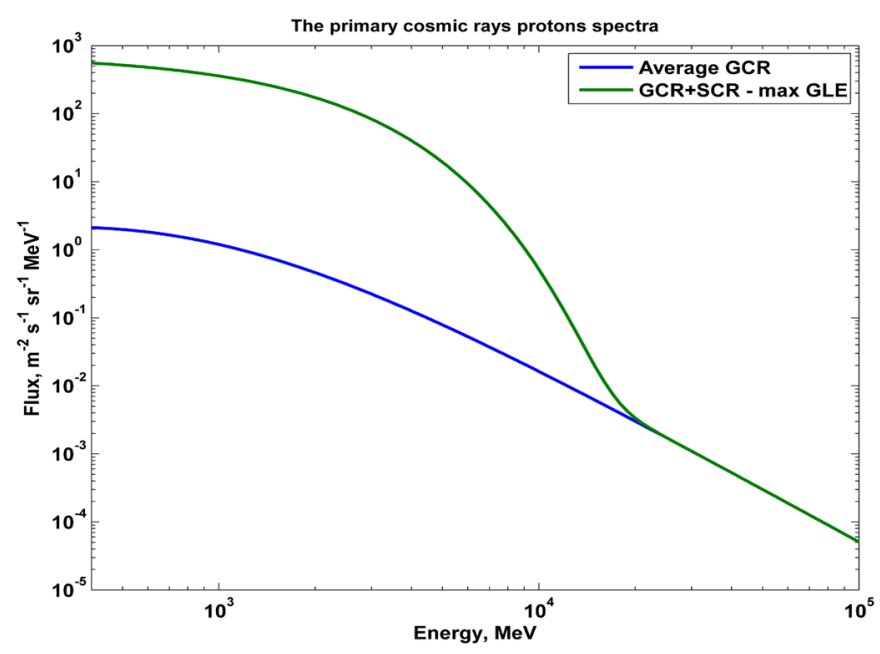

Fig. 1. Differential energy spectra of primary CR protons in the region from $400 \mathrm{MeV}$ to $100 \mathrm{GeV}$. The blue line are protons of GCRs and the green line are the protons of GCRs summed with those of SCRs, i.e. GCR protons of + SCR protons.

about a secondary CR cascades. The basis of the RUSCOSMICS software package is a GEANT4 program, which is used for class inheritance responsible for particles interaction with matter, setting initial parameters, gathering information on modeling, particle state, and others $[18,19]$.

\section{Ionization profiles in stratosphere and troposphere during GLE05}

As we already mentioned in the Introduction at present one of the most reliable systems for the registration of cosmic rays are the international network of ground-based neutron monitors (official database http://gle.oulu.fi/). According to this network the characteristics of SCRs recorded during the ground level enhancements are determined by solving the corresponding inverse problem [18, 19 and references therein]. Balloons are also traditionally launched.

One of a most difficult application in the RUSCOSMICS software package is a model for calculation of a primary $\mathrm{CR}$ protons transportation through the atmosphere and a secondary CR formation cascade study. To build it the "flat" geometry concept is applied. In this realization a column from whole Earth's atmosphere is created at a given latitude and longitude. Then layer parameters (density, pressure and temperature) are calculated with NRLMSISE-00 model [19].

A model particles (in our case protons) source is created on the upper bound with a spectral characteristic and intensity. A secondary CR cascade parameters are calculated with a primary CR transport trough an atmosphere matter. A model data can be compared with 
experimental ones obtained as a result of ballooning.

With RUSCOSMICS module a cosmic ray protons passage through the Earth's atmosphere was calculated both in a geophysical quiet periods, and for GLE events. The result of this work is the secondary CR energy spectra at different altitudes. These data can be used both for an ionization rate evaluation in a tasks of an equivalent dose calculation, and for a particle cascades formation detailed study to search a new features during GLE. Our simulation results were verified with a balloon experimental data and have a good agreement with it.

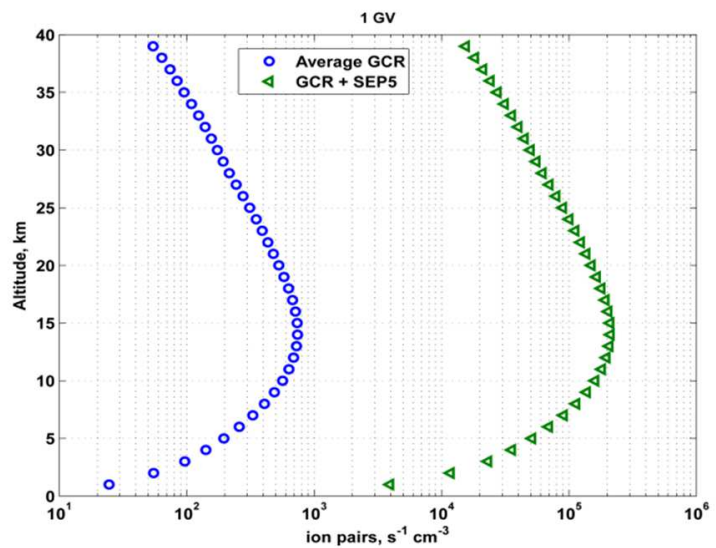

Fig. 2. Ion pairs profiles during GLE05 (or SEP05) on 23.02.1956 for geom. cut-off $1 \mathrm{GV}$.

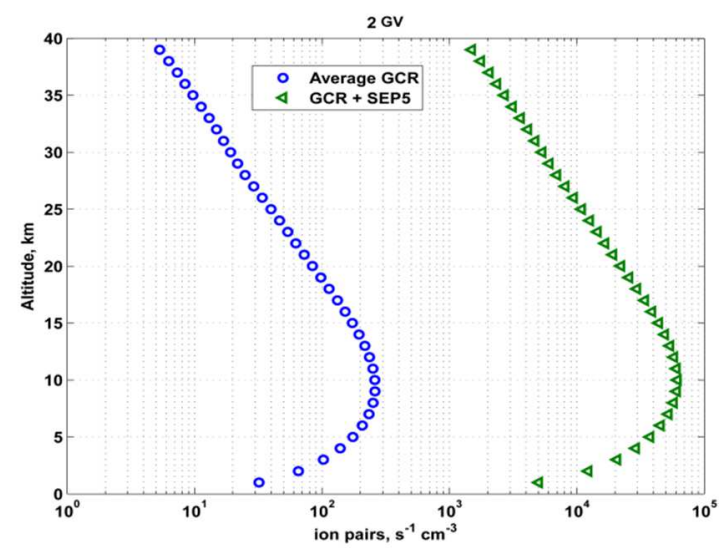

Fig. 3. Ion pairs profiles during GLE05 (or SEP05) on 23.02.1956 for geom. cut-off $2 \mathrm{GV}$.

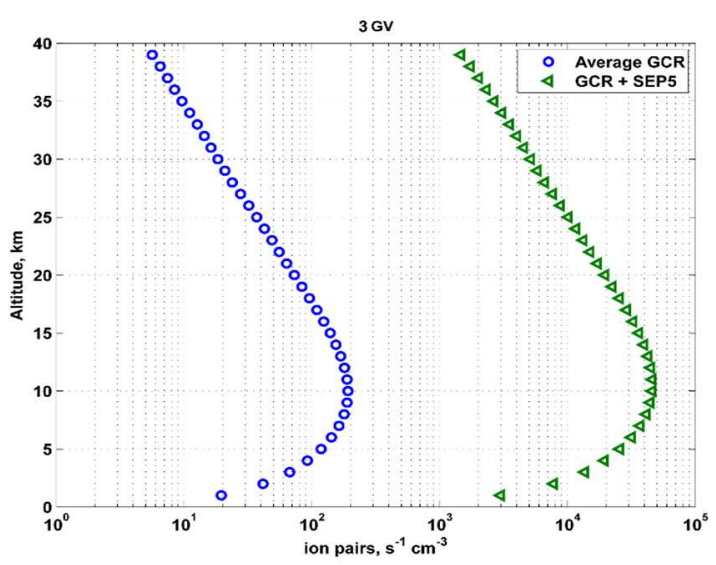

Fig. 4. Ion pairs profiles during GLE05 (or SEP05) on 23.02.1956 for geom. cut-off $3 \mathrm{GV}$.

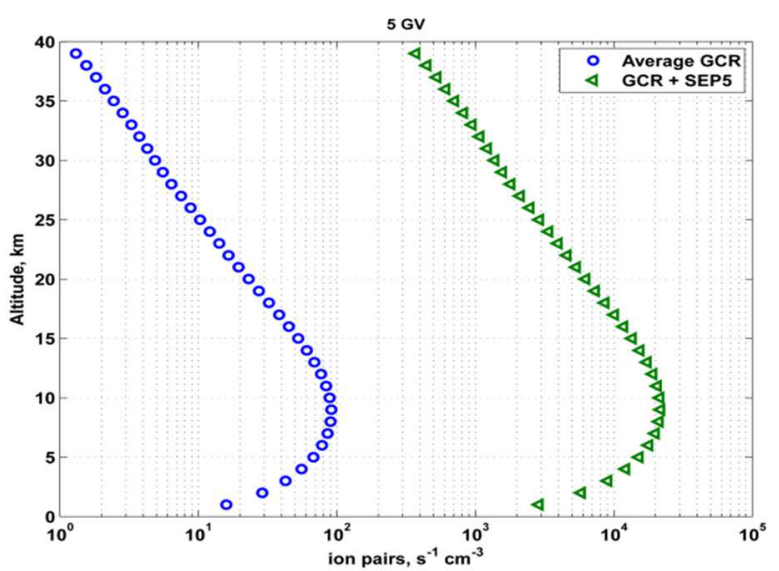

Fig. 5. Ion pairs profiles during GLE05 (or SEP05) on 23.02.1956 for geom. cut-off $5 \mathrm{GV}$.

The ion production rates during the GLE05 (i.e. SEP05) on 23 February 1956 are calculated as a superposition of ion rates due to SCRs and GCRs. The obtained ion production rate profiles due to GCRs and SEP05 at cut-off rigidity $\mathrm{Rc}=1 \mathrm{GV}$ are presented in Fig. 2. Accordingly, the ion production rate profiles due to GCRs and SEP05 for Rc $=2 \mathrm{GV}$ are plotted in Fig. 3, for Rc $=3 \mathrm{GV}$ on Fig. 4 and for Rc $=5 \mathrm{GV}$ in Fig. 5. As was expected the maximal ionization effect is observed in a low rigidity cut-off region, similarly to several previous work $[12,13,15,16]$, namely at polar and sub-polar regions (Figs. 2 and 3). At mid latitudes the ionization effect rapidly diminishes (Figs. 4 and 5). 


\section{Comparisson to other results}

The GCRs create the continuous and permanent ionization in the atmosphere, and also the independent ionospheric cosmic ray layer (CR layer) at altitudes $50-80 \mathrm{~km}$ in the D region in the lower ionosphere [5, 7-9]. Yet in [5, 7] are made some calculations of the electron production and electron density in the CR layer. These profiles were compared with the experimental profiles measured by Van Allen [5, 7 and references therein], on basis of data from German V2 rocket soundings in 1952 at geomagnetic latitudes $0^{\circ}, 38^{\circ}, 51^{\circ}$ and $60^{\circ}$. There was a satisfying coincidence between theoretical and experimental profiles with accuracy $\pm 10 \%$.

The results obtained in the present work (Figures 2-5) can also be compared with profiles of the Van Allen [5, 7 and references therein], but in the area 0-40 km. Here coincidence between computer simulations and experimental measurements of GCR ionization is accurate to within \pm $10-15 \%$. This difference may be due to incorporation of us concrete atmospheric model. It must be noted that such a comparison can be performed only for GCRs, because for SCRs such rocket measurements are missing.

\section{Discussion and conclusion}

The solar eruptive-flare event of 23 February 1956 is of big importance for the solar and solar-terrestrial community due to its outstanding characteristics. This event brought widespread attention to the Sun and cosmic ray observations, promoted the organization and success of cosmic radiation studies during the IGY (1957-1958) and led to the creation of the world-wide cosmic ray observatory network.

Actually the cosmic rays produce a cascade process in the atmosphere. This cascade affects the physical and chemical properties of the Earth's atmosphere, particularly the ion balance. Thus the cosmic rays are the dominant source of ionization and dissociation of air, which leads to changes in minor constituents (including the ozone) in the stratosphere and troposphere. In this connection the detailed study of the cosmic ray induced ionization is very important. One possible tool is based on Monte Carlo simulation of the cascade process in the atmosphere, precisely the energy deposit and afterwards the estimation of ion pair production.

The computed ion production rate allows the estimation the maximal ionization effect during the GLE05 event in the atmosphere as a function of the altitude above sea level. The obtained here ionizations are significant during the main phase of the GLE05 at polar regions, especially in the low stratosphere (Figs. 2 and 3). The ionization effect in the polar, sub-polar and middle latitude regions of the Earth is important during the whole event, especially in the region of the Regener-Pfotzer maximum (10-15 km). The ion production due to SEPs is greater than GCR in across the studied altitude range $0-40 \mathrm{~km}$ above sea level, because of the hard spectra of the solar particles (Fig. 1).

In studying of other cases of GLE it was received the opposite result: at middle and lower latitudes GCR produced more ion pairs compared to SEPs in the whole atmosphere during the event $[13,14]$. These particles had the steeper fall the SCR spectrum (greater $\gamma$, i.e. softer spectrum (2)). In these cases already at $5 \mathrm{GeV}$ the SCR flux becomes less than GCR flux $[15,16]$.

The obtained results are important for the improvement of the recent models of induced ionization and other effects due to cosmic rays [21-30], for the various atmospheric processes (atmospheric physics and chemistry - for instance the ozone production in stratosphere [31], the 
red sprites and global electric circuit between the ground and the ionosphere [32, 33], studies of solar-terrestrial influences on the space weather $[34,35])$ and for many other applications.

\section{References}

[1] Perez-Peraza J., A. Gallegos-Cruz, E. Vashenyuk, Yu. V. Balabin, Relativistic proton production at the Sun in the October 28th, 2003 solar event, Adv. Space Res. (2005) doi:10.1016/j.asr.2005.01082.

[2] Perez-Peraza J., E. Vashenyuk, A. Gallegos-Cruz, Yu. V. Balabin, L.I. Miroshnichenko, Relativistic proton at the Sun in the 20 January 2005 solar event, Adv. Space Res. 41 (2008) 947-954.

[3] Meyer, P., E.N. Parker, J.A. Simpson, Solar cosmic rays of 23 February 1956 and their propagation through interplanetary space, Phys. Rev. 104 (1956) 768-783.

[4] Vashenyuk E.V., Yu.V. Balabin, L.I. Miroshnichenko, Relativistic solar protons in the ground level event of 23 February 1956: the new study, Adv. Space Res. 41 (2008) 926-935.

[5] Velinov P.I.Y. An expression for the ionospheric electron production rate by cosmic rays. C.R. Acad. Bulg. Sci. 19 (1966), no 2, 109-112.

[6] Velinov P.I.Y. On electron production rates in the polar cap ionosphere due to solar cosmic rays. C.R. Acad. Bulg. Sci., 20 (1967) 1275-1278.

[7] Velinov P.I.Y. On ionization of the ionospheric D-region by galactic and solar cosmic rays. J. Atmos. Terr. Phys., 30 (1968) 1891-1905.

[8] Velinov P.I.Y. On ionization of lower ionosphere by cosmic rays. Geomagnetism and Aeronomy, $\mathbf{8}$ (1968) 448-456.

[9] Velinov P.I.Y. On enhanced ionization in lower ionosphere of polar cap due to solar corpuscular fluxes. Bull. Russ. Acad. Sci., Phys. 32 (1968) 1906-1909.

[10] Velinov P.I.Y., A. Mishev, L. Mateev, Model for induced ionization by galactic cosmic rays in the Earth atmosphere and ionosphere, Adv. Space Res. 44 (2009) 1002-1007.

[11] Mishev A., P.I.Y. Velinov, Influence of low energy hadron interaction models on the atmospheric ionization due to cosmic ray heavy nuclei, C.R. Acad. Bulg. Sci. 67 (2014) 843-854.

[12] Velinov P.I.Y., S. Asenovski, K. Kudela, J. Lastovicka et al. Impact of cosmic rays and solar energetic particles on the Earth's environment, J. Space Weath. Space Clim. 3 (2013) A14, 1-17.

[13] Mishev A., P.I.Y. Velinov, Ionzation rate profiles due to solar and galactic cosmic rays during GLE 59 Bastille day 14 July, 2000, C.R. Acad. Bulg. Sci., 68 (2015) 359-366.

[14] Mishev A., P.I.Y. Velinov, Ionization effect due to cosmic rays during Bastille Day Event (GLE 59) on short and mid time scales, C.R. Acad. Bulg. Sci., 69 (2016) 1479-1484.

[15] Mishev A., P.I.Y. Velinov, Contribution of cosmic ray nuclei of solar and galactic origin to atmospheric ionization during SEP event on 20.01.2005, C.R. Acad. Bulg. Sci. 65 (2012) 373-380.

[16] Mishev A., P.I.Y. Velinov, A maverick GLE 70 in solar minimum. Calculations of enhance ionization in the atmosphere due to relativistic SEPs. C.R. Acad. Bulg. Sci. 66 (2013) 1457-1462.

[17] Mishev A., P.I.Y. Velinov, Determination of medium time scale ionization effects at various altitudes in the stratosphere and troposphere during ground level enhancement due to solar cosmic rays on 13.12.2006 (GLE 70), C.R. Acad. Bulg. Sci. 68 (2015) 1427-1432. 
[18] Mauchev E.A., Yu. V. Balabin, B.B. Gvozdevskii, E.V. Vashenyuk, A new numerical model for investigating CRs in the Earth's atmosphere, Bull. Russ. Acad. Sci., Phys., 79 (2015) 657-659.

[19] Mauchev E. A., Yu. V. Balabin, A practical application of RUSCOSMICS software package in a cosmic rays physics, Ed. I.V. Karpov: Proceedings of V International conference "Atmosphere, ionosphere, safety” (2016) Immanuel Kant University Press, Kaliningrad, pp. 384-388.

[20] Maurchev E.A., Yu.V. Balabin, E.V. Vashenyuk, B.B. Gvozdevsky, Transport of solar protons through the atmosphere during GLE, J. Phys. Conf. Ser. 409 (2013) 012200.

[21] Umahi A.E. Influence of galactic and solar cosmic rays on ionization in the atmosphere, IOSR Journal of Applied Physics (IOSR-JAP), 8 (2016) no. 4, Ver. II, 38-46.

[22] Umahi A.E. Galactic and solar cosmic rays on ionization in the atmosphere, World Applied Sciences Journal 34 (2016), no. 3, 312-317.

[23] Umahi, A.E. Earth's environmental pollution from galactic cosmic rays flux, World Applied Science Journal, 34 (2016) 338-342.

[24] Umahi, A.E. Impact of high energy charged galactic particle variations in the Earth's atmosphere, Middle-East Journal of Scientific Research, 24 (2016) 1788-1793.

[25] Umahi, A.E. Effects of cosmic rays and solar flare variations in earth's atmospheric mechanism and ionization, Middle-East Journal of Scientific Research, 24 (2016) 1794-1801.

[26] Umahi, A.E., Solar modulation on galactic cosmic rays in the Earth's atmosphere, IOSR Journal of Applied Physics (IOSR-JAP) 8 (2016) no. 4, Ver. II, 32-37.

[27] Umahi, A.E., P.A. Okpara, D.N. Oboma, V.N. Udeaja et al. On the dynamics of galactic cosmic rays in the atmosphere, IOSR Journal of Environmental Science (IOSR-JESTFT) 10 (2016) no. 7, 80-84.

[28] Umahi, A.E. (2016) Impact of space radiation in the Earth's atmosphere, American-Eurasian J. Agric. \& Environ. Sci., 16 (5), 868-873.

[29] Umahi, A.E. (2016) Variability of galactic cosmic rays flux and solar activities in the Earth's atmospheric environment, American-Eurasian J. Agric. \& Environ. Sci., 16 (5), 874-881.

[30] Balabin Yu.V., D.D. Dzhappuev, B.B. Gvozdevsky, E.A. Maurchev, A.U. Kudzhaev, O.I. Mikhailova, Neutron multiplicity: Local hadronic showers and extensive air showers, Bull. Russ. Acad. Sci., Phys. 75 (2011) 364-366.

[31] Kilifarska N. An autocatalytic cycle for ozone production in the lower stratosphere initiated by galactic cosmic rays, C. R. Acad. Bulg. Sci. 66 (2013) 243-252.

[32] Tonev P. Influence of solar activity on dimensions of red sprites caused by long-term variations of strato-mesospheric conductivity - model study, C.R. Acad. Bulg. Sci. 70 (2017) 111-120.

[33] Tonev P. Estimation of currents in global atmospheric electric circuit with account of transpolar ionospheric potential, C.R. Acad. Bulg. Sci. 65 (2012) 1593-1602.

[34] Abunina M., A. Abunin, A. Belov, S. Gaidash, Y. Tassev, P.I.Y. Velinov, L. Mateev, P. Tonev, Propeties of magnetic fields in coronal holes and geoeffective disturbances in solar cycle 24, C.R. Acad. Bulg. Sci. 67 (2014) 699-704.

[35] Mateev L., Y. Tassev, P.I.Y. Velinov, Application of the idea of morphism in solar-terrestrial physics and space weather, C. R. Acad. Bulg. Sci. 69 (2016) 1621-1630. 